\title{
Anisotropic shock response of columnar nanocrystalline $\mathrm{Cu}$
}

\author{
Sheng-Nian Luo, ${ }^{1, a)}$ Timothy C. Germann, ${ }^{1}$ Tapan G. Desai, ${ }^{2}$ Davis L. Tonks, ${ }^{1}$ and \\ Qi $A n^{1,3}$ \\ ${ }^{1}$ Los Alamos National Laboratory, Los Alamos, New Mexico 87545, USA \\ ${ }^{2}$ Advanced Cooling Technologies Inc., Lancaster, Pennsylvania 17601, USA \\ ${ }^{3}$ Materials and Process Simulation Center, California Institute of Technology, \\ Pasadena, California 91125, USA
}

(Received 30 March 2010; accepted 4 May 2010; published online 16 June 2010)

\begin{abstract}
We perform molecular dynamics simulations to investigate the shock response of idealized hexagonal columnar nanocrystalline $\mathrm{Cu}$, including plasticity, local shear, and spall damage during dynamic compression, release, and tension. Shock loading (one-dimensional strain) is applied along three principal directions of the columnar $\mathrm{Cu}$ sample, one longitudinal (along the column axis) and two transverse directions, exhibiting a strong anisotropy in the response to shock loading and release. Grain boundaries (GBs) serve as the nucleation sites for crystal plasticity and voids, due to the GB weakening effect as well as stress and shear concentrations. Stress gradients induce GB sliding which is pronounced for the transverse loading. The flow stress and GB sliding are the lowest but the spall strength is the highest, for longitudinal loading. For the grain size and loading conditions explored, void nucleation occurs at the peak shear deformation sites (GBs, and particularly triple junctions); spall damage is entirely intergranular for the transverse loading, while it may extend into grain interiors for the longitudinal loading. Crystal plasticity assists the void growth at the early stage but the growth is mainly achieved via GB separation at later stages for the transverse loading. Our simulations reveal such deformation mechanisms as GB sliding, stress, and shear concentration, GB-initiated crystal plasticity, and GB separation in nanocrystalline solids under shock wave loading. (C) 2010 American Institute of Physics. [doi:10.1063/1.3437654]
\end{abstract}

\section{INTRODUCTION}

Defects, impurities and interfaces are ubiquitous in a real solid (even in a "single crystal"), and can be equally important as its crystal structure regarding the mechanical and physical properties under various loading conditions. This microstructure effect is a key and most active area in materials physics. ${ }^{1}$ Dynamic materials response, such as plasticity and spall damage induced by shock wave loading, is often strongly dependent on microstructure; e.g., the shock experiments exploring the microstructure effect on spall damage are reviewed by Meyers and Aimone $^{2}$ as well as Kanel. ${ }^{3}$ Molecular dynamics (MD) simulations are advantageous in revealing the materials response and underlying new phenomena arising from particular microstructures. Despite inherent limitations, chiefly in the accessible timescale (typically nanosecond), such simulations are first-principlesbased (with particularly reliable force fields for close-packed metals) and can access relevant spatial resolutions. Given the importance of grain boundaries (GBs), considerable efforts including MD simulations have been dedicated to the mechanical or physical properties of different nanostructures under nonshock conditions. ${ }^{4-15} \mathrm{MD}$ is also a proven tool in modeling shock response and providing valuable physical insights. ${ }^{16-24}$ However, MD shock simulations of defective solids $^{21-23,25}$ are few and focus on nanocrystalline metals with random GBs. We recently investigated with MD simulations the shock response of perfect $\mathrm{Cu}$ and $\mathrm{Cu}$ with differ-

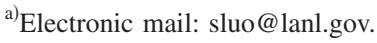

ent vacancy concentrations, ${ }^{20,25}$ and performed shock experiments on multicrystalline $\mathrm{Cu},{ }^{26,27}$ along with continuum scale modeling. ${ }^{28} \mathrm{MD}$ and molecular statics were utilized to model the plastic deformation and fracture in columnar nanocrystalline $\mathrm{Ni}$ and $\mathrm{Al}$ under nonshock loading. ${ }^{10-12} \mathrm{As}$ a natural extension of our earlier shock wave studies, it is highly desirable to examine GBs in a geometry much simpler than abundant random GBs, in order to elucidate some elemental processes and mechanisms in shock response. We choose an idealized hexagonal columnar nanocrystalline $\mathrm{Cu}$ as a model system to explore in detail its shock response, to connect GBs and triple junctions to plasticity, local shear, and spall damage as well as their spatial heterogeneities and anisotropy, and to reveal the deformation mechanisms under shock loading. Sec. II addresses the methodology for MD simulations and analysis. The results and discussion are presented in Sec. III followed by conclusions in Sec. IV.

\section{METHODOLOGY}

The atomic interactions in $\mathrm{Cu}$ are described with an accurate embedded-atom-method potential by Mishin et al. ${ }^{29}$ Our MD simulations are performed using the Institut für Theoretische und Angewandte Physik (ITAP) MD (IMD) code. ${ }^{30}$ We first construct an idealized hexagonal columnar nanocrystalline microstructure unit with the Voronoi tessellation method. ${ }^{7,8,31}$ It contains six grains of identical hexagonal shape and diameter within the three-dimensional (3D) periodic cell. We choose a grain diameter of about $13 \mathrm{~nm}$ for exploring the GB void nucleation under given loading conditions. The columnar axis is along [100] (the rotation axis), 


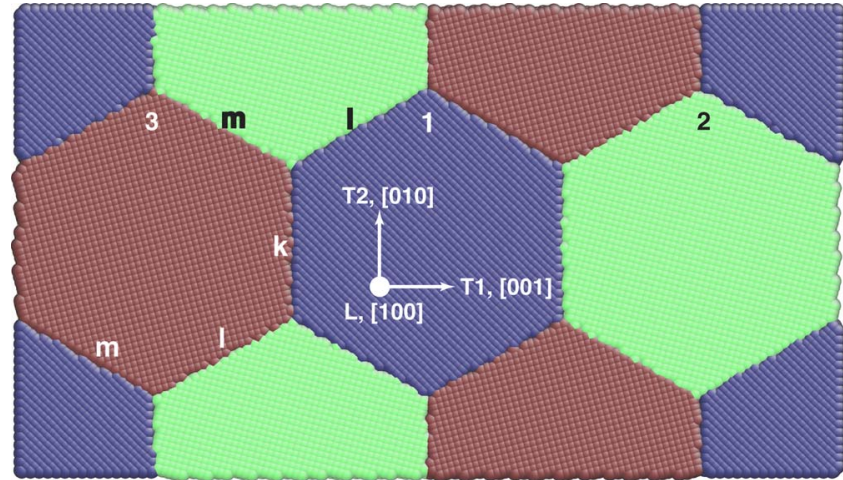

FIG. 1. (Color online) The configuration of a six-grain columnar $\mathrm{Cu}$ structure unit, viewed along the rotation axis [100]. It also represents the crosssection for the $L$-direction loading. Numbers 1-3 denote grain types with different color coding.

and the thickness along this direction is about $7.2 \mathrm{~nm}$. Grain type 1, defined in Fig. 1, is the reference grain for rotation; the rotation angle is $60^{\circ}$ and $30^{\circ}$ for types 2 and 3, respectively. For each grain type, there are two identical grains in the periodic cell, and all the neighboring grains are of different grain types. The construction yields only high energy, asymmetric GBs (the misorientation angle is $30^{\circ}$ ). The resulting microstructure unit ( 400 000 atoms) is relaxed with the conjugate gradient method, followed by thermalization at the ambient conditions with the constant-pressuretemperature $(N P T)$ ensemble and 3D periodic boundary conditions.

Each hexagon has three pairs of parallel sides $(k-m$, Fig. 1). Sides $k-m$ of three neighboring grains form a Y-shape (GB-Y). The transverse ( $T 1$ and $T 2$ ) and longitudinal $(L)$ directions (Fig. 1) define the coordinate systems of our simulations. Each of these directions is chosen as the shock loading axis and denoted as the $x$-axis, and the other two, the $y$-axis and $z$-axis, respectively. The flyer plate-target configuration is adopted for planar shock-spall simulations. ${ }^{16,32}$ To construct the targets for the T1-direction, T2-direction, and $L$-direction shock loading, the microstructure unit is replicated by 2, 4, and 12 times along the respective directions (Figs. 1 and 2); the corresponding dimensions are about 68 $\times 20 \times 7 \mathrm{~nm}^{3}, \quad 80 \times 34 \times 7 \mathrm{~nm}^{3}$, and $86 \times 34 \times 20 \mathrm{~nm}^{3}$ (containing $\sim 800000,1600000$, and 4800000 atoms, respectively). The flyer plates have the same cross-sectional areas as the corresponding targets but are reduced in thickness by half along the shock or $x$ direction. The flyer plates and targets are further equilibrated with the NPT ensemble under ambient conditions, with 3D periodic boundary conditions.

A flyer plate and the corresponding target are assembled along the $x$-axis. We denote the supported shock state particle velocity (or equivalently, the piston velocity) as $u_{\mathrm{p}}$. The flyer plate and target are assigned initial velocities of $4 / 3 u_{\mathrm{p}}$ and $-2 / 3 u_{\mathrm{p}}$ along the $x$-axis, respectively, before impacting each other; $u_{\mathrm{p}} \approx 0.48 \mathrm{~km} \mathrm{~s}^{-1}$ is chosen here, at which the $\mathrm{Cu}$ single crystals remain elastic upon shock while the columnar $\mathrm{Cu}$ shows plasticity due to GBs. ${ }^{20,32}$ Shock simulations adopt the microcanonical NVE ensemble. ${ }^{16,32}$ Periodic boundary conditions are applied along the $y$-axis and $z$-axis (not the

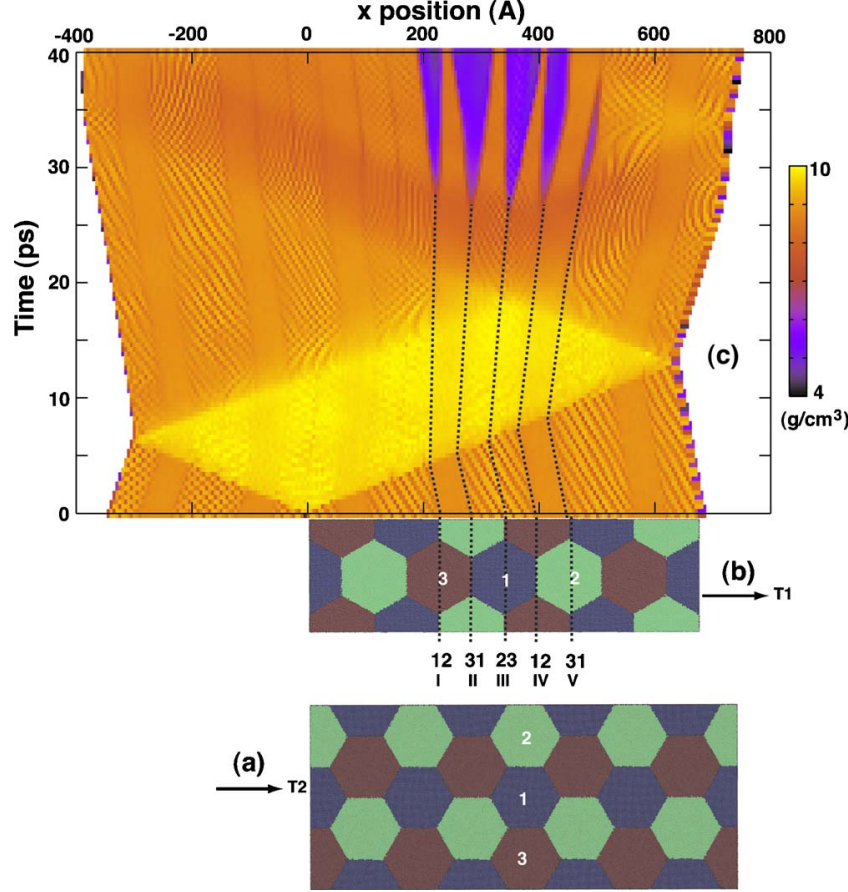

FIG. 2. (Color online) The configurations for shock loading along the $T 2$-direction (a) and T1-direction (b), and the $x-t$ diagram for the loading along the T1-direction (c). Color coding in (c) is based on the local density $\rho(x)$. The schematic dashed lines indicate the $k$-type GBs I-V. The number pair 12 denotes the GB between type 1 and 2 grains (similar for others).

$x$-axis) to mimic one-dimensional (1D) strain loading. The nonimpact sides of the flyer plate and target are free surfaces. The time step for integrating the equations of motion is $1 \mathrm{fs}$, and the shock run durations are up to $45 \mathrm{ps}$.

Neglecting the heterogeneities normal to the shock direction (i.e., on the cross-sections), we divide the simulation cell into fine bins only along the $x$-direction (the 1D binning analysis $^{32}$ ), and the average physical properties are obtained within each bin, such as density $(\rho)$, stress $\left(\sigma_{i j}\right)$, particle velocity $(u)$, and temperature $(T)$ profiles along the $x$-axis at different stages of compression, release, and tension. The center-of-mass velocity of a bin is removed when calculating $T$ and $\sigma_{i j}$ from the decomposed virial method. ${ }^{33}$ The free surface velocity $\left(u_{\mathrm{fs}}\right)$ vs. time $(t)$ is obtained from the particle velocity evolution on the target free surface. Similarly, twodimensional (2D) and 3D binning analyses (i.e., including binning along the $y$-axis or $z$-axis as well) are performed to better resolved spatially GBs and different grains.

We characterize the local deformation and local structure around an atom with the local von Mises shear strain $\left(\eta^{\mathrm{vM}}\right)$ (Refs. 25 and 34 ) and centrosymmetry parameter, ${ }^{35}$ both useful for visualizing crystal plasticity. $\eta^{\mathrm{vM}}$ may reveal shear slip bands within a grain and GB sliding, and centrosymmetry, resolve different atomic packing orders: original facecentered-cubic (fcc) packing, stacking faults and twins in hexagonal-close-packed (hcp) packing and other defects in nonclose-packed structure. Atoms in the slip bands are normally in the hcp packing during plastic deformation of $\mathrm{Cu}$, thus a manifestation of crystal plasticity. ${ }^{25}$

The shock state or Hugoniot state (denoted with a subscript $\mathrm{H})$ parameters are obtained from the 1D profiles. We 


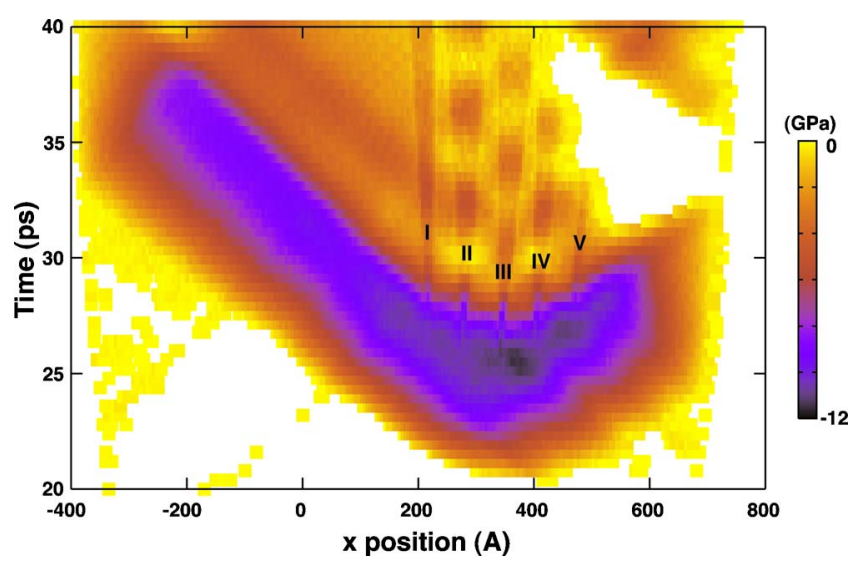

FIG. 3. (Color online) The $x-t$ diagram for the $T 1$-direction loading. Color coding is based on the local stress $\sigma_{x x}(x)$.

define $\tau$ as the maximum shear stress, and $2 \tau=\sigma_{x x}-\left(\sigma_{y y}\right.$ $\left.+\sigma_{z z}\right) / 2$. For plastic deformation during compression or tension, the von Mises shear flow stress or strength can be deduced from the evolution of $|2 \tau|$ in 1D-3D profiles. The spall temperature $\left(T_{\mathrm{sp}}\right)$, tensile strain rate, and spall strength $\left(\sigma_{\mathrm{sp}}\right.$, i.e., the maximum tensile stress $-\sigma_{x x}$ ) are evaluated in situ right before spallation near the spall zone from 1D (2D or 3D) analysis. ${ }^{32}$

\section{RESULTS AND DISCUSSION}

1D analysis of shock loading is of practical use in interpreting many experimental measurements and continuum mechanics modeling, and are thus presented here first. Physical properties are only resolved spatially along the shock or $x$ direction in the 1D analysis. The density evolution for the $T 1$-direction loading, plotted in a traditional $x-t$ (positiontime) diagram, illustrates the wave propagation and interaction such as: the impact-induced shocks, the subsequent release fans originating at free surfaces, and the interaction of the opposing release fans, which yield well defined shock, release, tensile, and spall regimes [Fig. 2(c)]. The corresponding longitudinal stress evolution is shown in Fig. 3 (only tensile states are shown). Upon spall, the tensile stress is reduced, inducing (re-)compression waves propagating toward the free surfaces. Spall reduces local tension (Fig. 3) and raises the local temperature. This shock-release-spall sequence is also manifested in the free surface velocity history (Fig. 4). In particular, the recompression following spall is registered in $u_{\mathrm{fs}}(t)$ as a pullback characteristic of spallation.

The elastic-plastic or two-wave structure upon shock compression is observed within the target or on the free surface (Fig. 4). The plastic shock plateaus for the T1-direction and $T 2$-direction show fluctuations due to the grain structures along the shock directions. The shock state $\sigma_{x x, \mathrm{H}}$ and $T_{\mathrm{H}}$ are obtained in situ and listed in Table I. $\sigma_{x x, \mathrm{H}}$ decreases slightly in the order of the T1-direction, T2-direction, and $L$-direction, likely due to the slight differences in the effective wave speeds. $T_{\mathrm{H}}$ is similar for all three directions but slightly higher for the transverse loading, largely due to the more pronounced GB sliding (see below). The apparent plasticity is a result of crystal plasticity and GB sliding or geometric accommodation of different grains as shown below.

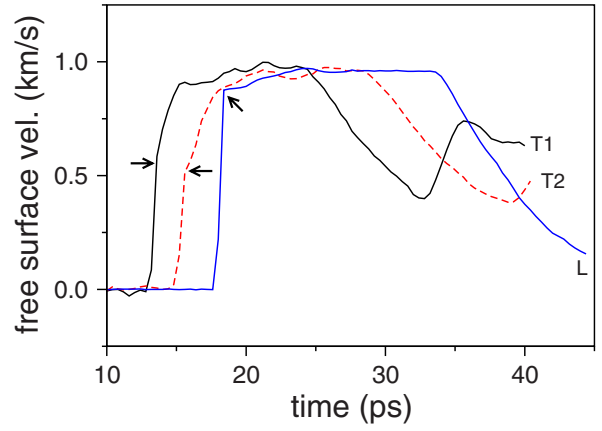

FIG. 4. (Color online) Free surface histories for loading along three different directions $(T 1, T 2$, and $L)$. The arrows indicate the HEL. Spall pullback in $u_{\mathrm{fs}}(t)$ is evident for the transverse $T 1$-direction and $T 2$-direction loading.

The Hugoniot elastic limit (HEL) as identified from $u_{\mathrm{fs}}(t)$ increases in the order of the $T 2$-direction, $T 1$-direction, and $L$-direction if we neglect the difference in elastic wave speed. However, the flow stress is a better quantity and can be directly obtained from our simulations as $2 \tau_{\max }$ in the elastic region (Table I); it decreases in the order of the $T 1$-, $T 2$ - and $L$-directions (higher for the transverse loading). Compared to single crystal $\mathrm{Cu}$, the flow stress of columnar $\mathrm{Cu}$ is reduced by different amounts due to the GB geometry and plasticity anisotropy. For example, no crystal plasticity is observed in shocked $\langle 100\rangle$ single crystal and its $2 \tau_{\max }$ is above $4.6 \mathrm{GPa}^{25}$ while pronounced plasticity occurs for the $L$-direction loading, and $2 \tau_{\max }$ is reduced to about $4 \mathrm{GPa}$. We need to point out that the shape and amplitude of the elastic precursor and plastic plateau depend on the propagation distance (and to a lesser extent, the number of grains on the cross-sections). Ideally, the dimensions of the flyer and target should be extended substantially for a more complete picture of the elastic-plastic transition. However, such computations are prohibitively expensive for conventional MD. The relevant values reported in Table I are thus not intended to be exact.

Spallation is manifested as, e.g., pronounced pullbacks in $u_{\mathrm{fs}}(t)$ (Fig. 4) or low density zones in the $x-t$ diagram in terms of $\rho(x)$ [Fig. 2(c)] for all three loading directions. The $x-t$ diagrams [e.g., Figs. 2(c) and 3] show narrow, periodically spaced "spall planes" for the T1-direction and T2-direction loading, and broad, irregularly spaced spall zones for the $L$-direction loading. The periodicity in spall planes for the T1-direction is exactly the spacing between neighboring $k$-type GBs [Figs. 2(b) and 2(c)], and it is about the spacing of $l$-type or $m$-type $\mathrm{GBs}$ for the $T 2$-direction loading [Fig. 2(a)]. GBs are uniform along the shock direction for the $L$-direction loading so the spall zones are broad.

The maximum tensile stress from the 1D stress profiles, $\sigma_{x x}(x)$, is normally taken as $\sigma_{\mathrm{sp}}{ }^{24,32}$ This method may work well for single crystals or if the structure variation along the shock direction can be neglected, e.g., the columnar $\mathrm{Cu}$ subjected to the $L$-direction loading. However, it may yield misleading results since the location with the maximum tensile stress may be stronger and undergo no spallation, while spall occurs at weak regions even at lower tensile stresses, as illustrated in the case of the T1-direction loading (Fig. 3). We thus evaluate the maximum tensile stress at individual spall 
TABLE I. Shock and spall parameters of columnar nanocrystalline $\mathrm{Cu}$ from 1D analysis.

\begin{tabular}{lccccc}
\hline \hline Load direction & $\begin{array}{c}\sigma_{x x, \mathrm{H}} \\
(\mathrm{GPa})\end{array}$ & $\begin{array}{c}2 \tau_{\max } \\
(\mathrm{GPa})\end{array}$ & $\begin{array}{c}T_{\mathrm{H}} \\
(\mathrm{K})\end{array}$ & $\begin{array}{c}T_{\mathrm{sp}} \\
(\mathrm{K})\end{array}$ & $\begin{array}{c}\sigma_{\mathrm{sp}} \\
(\mathrm{GPa})\end{array}$ \\
\hline$T 1$ & 21.3 & 6.2 & 430 & 340 & $10.7-11.2$ \\
$T 2$ & 20.8 & 5.6 & 430 & 340 & $11.2-11.7$ \\
$L$ & 20.6 & 4.0 & 415 & 300 & 13.8 \\
\hline \hline
\end{tabular}

planes for the T1-direction and T2-direction loading. The values of $\sigma_{\mathrm{sp}}$ are listed in Table I along with $T_{\mathrm{sp}} . \sigma_{\mathrm{sp}}$ is similar for the $T 1$-direction and $T 2$-direction (the former is slightly weaker), but considerably lower than for the $L$-direction. Note stress concentration is observed in the transverse loading (Fig. 3) but not in the longitudinal loading from the 1D stress profiles. Spall is expected to occur more easily at GBs that are normal to or at a finite angle with the tensile stress, as for the T1-direction and T2-direction. GBs weaken the columnar $\mathrm{Cu}$ regardless of the loading directions, since voids nucleate preferentially at the inherently weaker GBs for the grain size and loading conditions examined here (see below). For instance, no spallation occurs in shocked $\langle 100\rangle$ single crystal at similar $u_{\mathrm{p}}$ (the tensile stress is above $16 \mathrm{GPa}),{ }^{25}$ while $\sigma_{\mathrm{sp}}$ is reduced to about $13.8 \mathrm{GPa}$ for the $L$-direction loading. $T_{\mathrm{sp}}$ is also higher for the transverse loading, likely due to the pronounced GB shear or sliding (see below). The tensile strain rate estimated in situ from $u(x)$ is about $10^{9}-10^{10} \mathrm{~s}^{-1}$.

The 1D analysis is not adequate or accurate as regards the shock response of columnar nanocrystalline $\mathrm{Cu}$. For example, Figs. 2(b) and 2(c) clearly show that spall occurs at $k$-type GBs for the T1-direction loading, while the spall stress deduced from 1D analysis is the average of those for the GBs and the grain interiors. 1D analysis smears spallation (as well as plastic deformation) features due to its lack of spatial resolutions on the cross-sections normal to the shock direction. For shock loading along the T1-direction and $T 2$-direction, the spatial variations in physical properties along the columnar axis can be neglected, so $2 \mathrm{D}$ analysis is sufficient (i.e., averaging over the $L$-direction). For the $L$-direction loading, $3 \mathrm{D}$ analysis is necessary since there are stress gradients along the shock direction and spatial heterogeneities in the orthogonal directions. Figures 5-14 presents the results from the 2D and 3D analyses in terms of local stress, temperature, shear, potential energy and structure.

When subjected to shock compression at similar $u_{\mathrm{p}}$, plastic deformation and spatial variations in physical properties on the cross-sections are negligible in the single crystal $\mathrm{Cu}$ for a variety of loading directions. ${ }^{32}$ In sharp contrast, crystal plasticity or deformation heterogeneities are well manifested in columnar nanocrystalline $\mathrm{Cu}$ (Figs. 5-10). The centrosymmetry analysis (Figs. 8-10; visualization adopts AtomEye $^{36}$ ) shows that crystal plasticity initiates at GBs and occurs as changes in atomic packing from fcc to hcp ordering and other defects, e.g., dislocation cores. Both stacking faults and twins are observed in the hcp packing. (The former involves two adjacent hep layers or two hcp layers separated by a fcc layer, and the latter, one hcp layer.) The elevated free energy of GBs reduces the energy barrier to plasticity nucleation; GB free volume and local stress concentration may also play a role. ${ }^{4}$ Similarly, we observed the enhancement of crystal plasticity by vacancies during shock loading. ${ }^{25}$ Such nucleation sites are absent in single crystal $\mathrm{Cu}$ so it remains essentially elastic at similar loading conditions.

In the case of the $T 1$-direction and T2-direction loading, the loading direction for type 1 grains is $\langle 100\rangle$, while it is rotated by $\pm 30^{\circ}$ for type 2 and 3 grains, and thus the slip planes and directions are rotated accordingly. As a result,

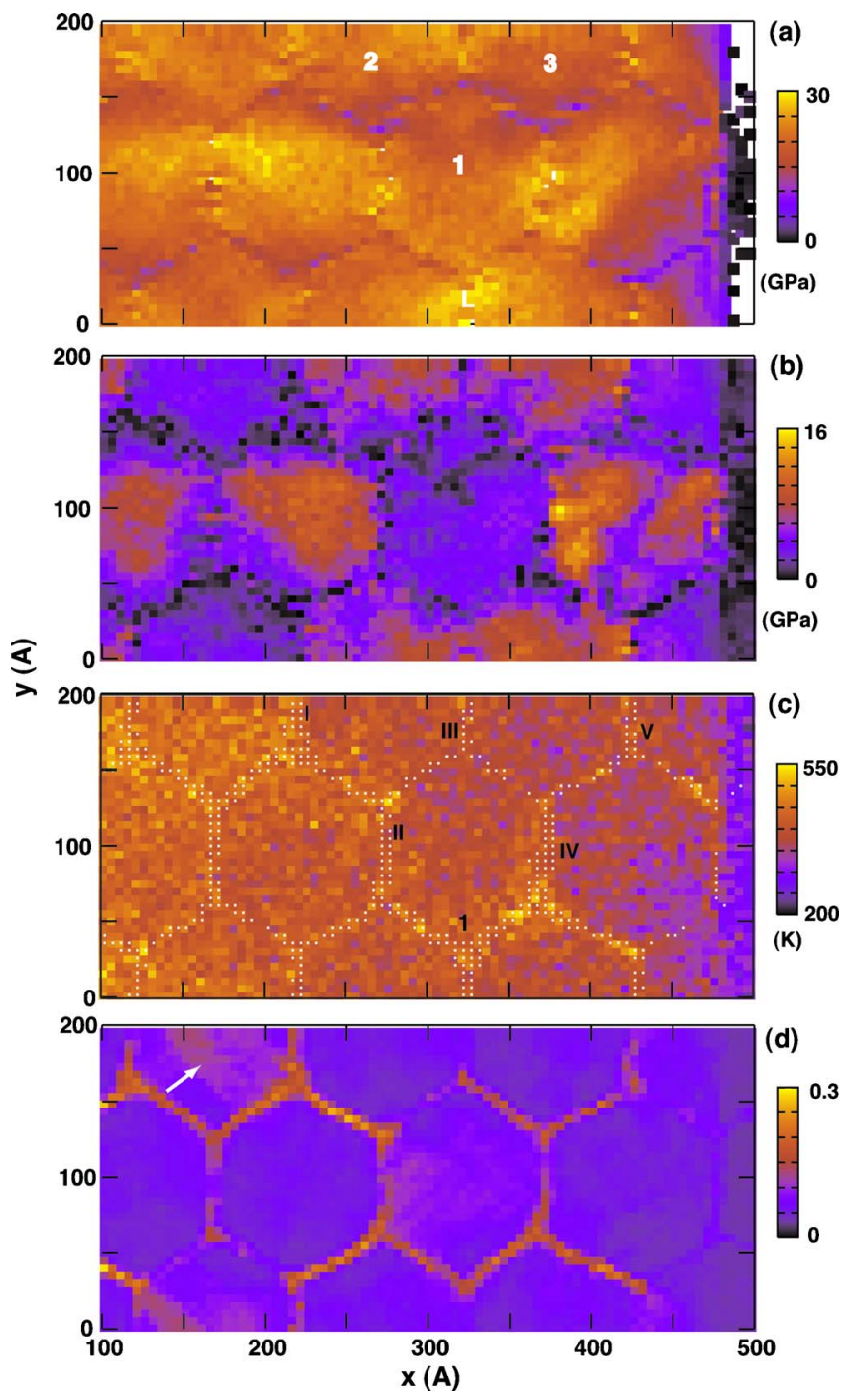

FIG. 5. (Color online) 2D distribution of $\sigma_{x x}(\mathrm{a}),|2 \tau|$ (b), temperature (c), and $\eta^{\mathrm{vM}}(\mathrm{d})$, averaged over the $L$-direction for the $T 1$-direction loading at $t=10 \mathrm{ps}$ (shock compression). The $k$-type GBs (I-V) and representative grains (1-3) are indicated. The white dots in (c) denote the GB profile. The arrow in (d) denotes a slip band within a grain interior. 


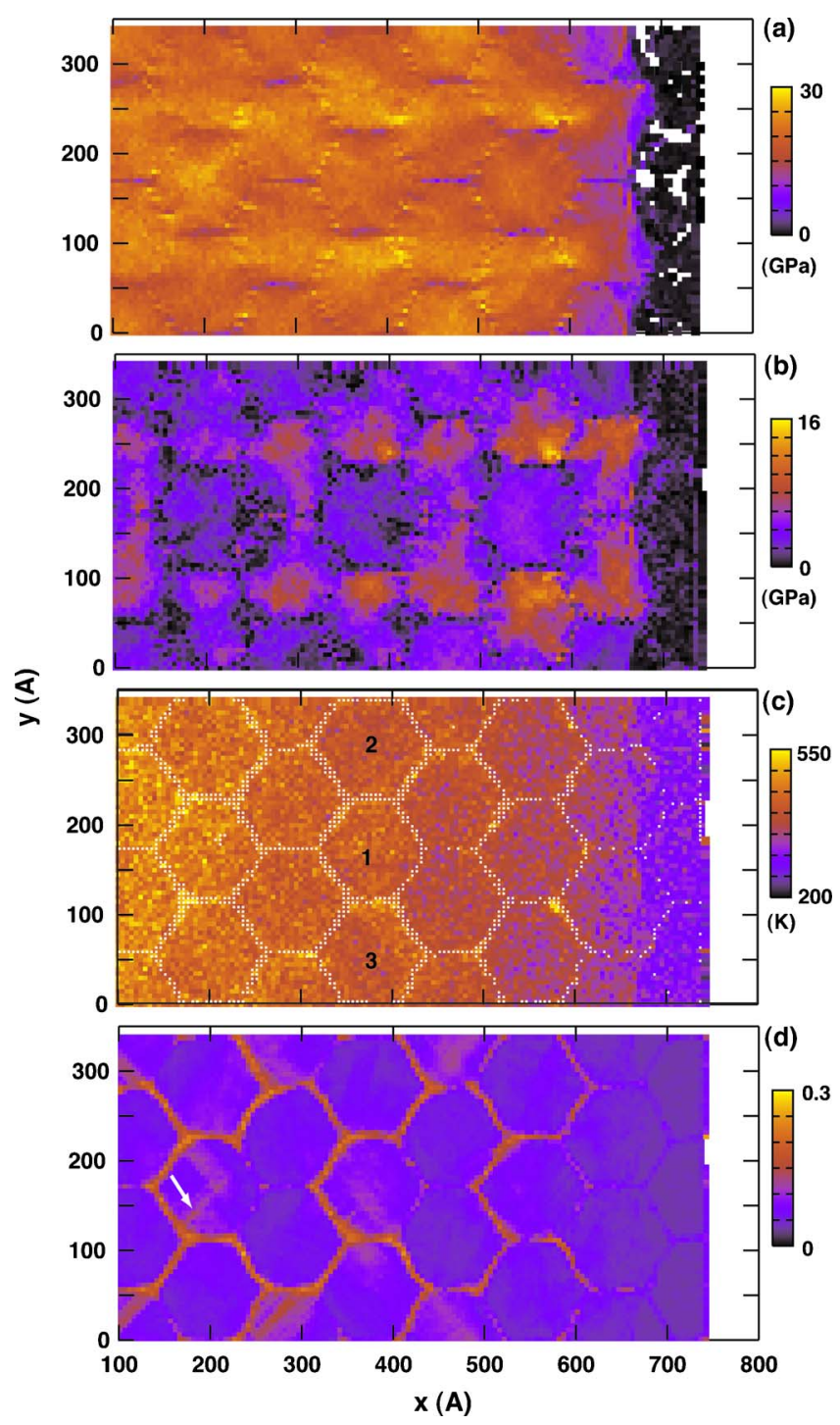

FIG. 6. (Color online) 2D distribution of $\sigma_{x x}$ (a), $|2 \tau|$ (b), temperature (c), and $\eta^{\mathrm{vM}}(\mathrm{d})$, averaged over the $L$-direction for the $T 2$-direction loading at $t=14$ ps (shock compression). In (c), numbers 1-3 indicate three typical grains, and the GB profile is indicated with white dots. The arrow in (d) denotes a slip band.

crystal plasticity in type 1 grains is more pronounced than in type 2 and 3 grains (Figs. 8 and 9), due to the different Schmid factors for different grain orientations. This is evident in the first frame of Fig. 9, as the active $\{111\}$ slip systems in type- 1 grains are much closer to the optimal $45^{\circ}$ inclinement to the shock direction than either type- 2 or 3 grains. The $T 2$-direction loading yields slightly more plasticity than the $T 1$-direction loading, a manifestation of the orientation effect of the "GB-Y." In the case of the $L$-direction loading, all the grains are identical with respect to the loading direction and essentially the type 1 grains in the $T 1$ - and T2-direction loading; therefore, the plasticity is marked and "uniform" among different grains [Fig. 10(a)]. Overall, crystal plasticity increases in the order of the $T 1-, T 2$-direction and $L$-direction loading, consistent with the decrease in flow stress obtained from the 1D analysis (Table I).

Local shear deformation (Figs. 5-7) is also characteristic of heterogeneous and anisotropic response of the columnar

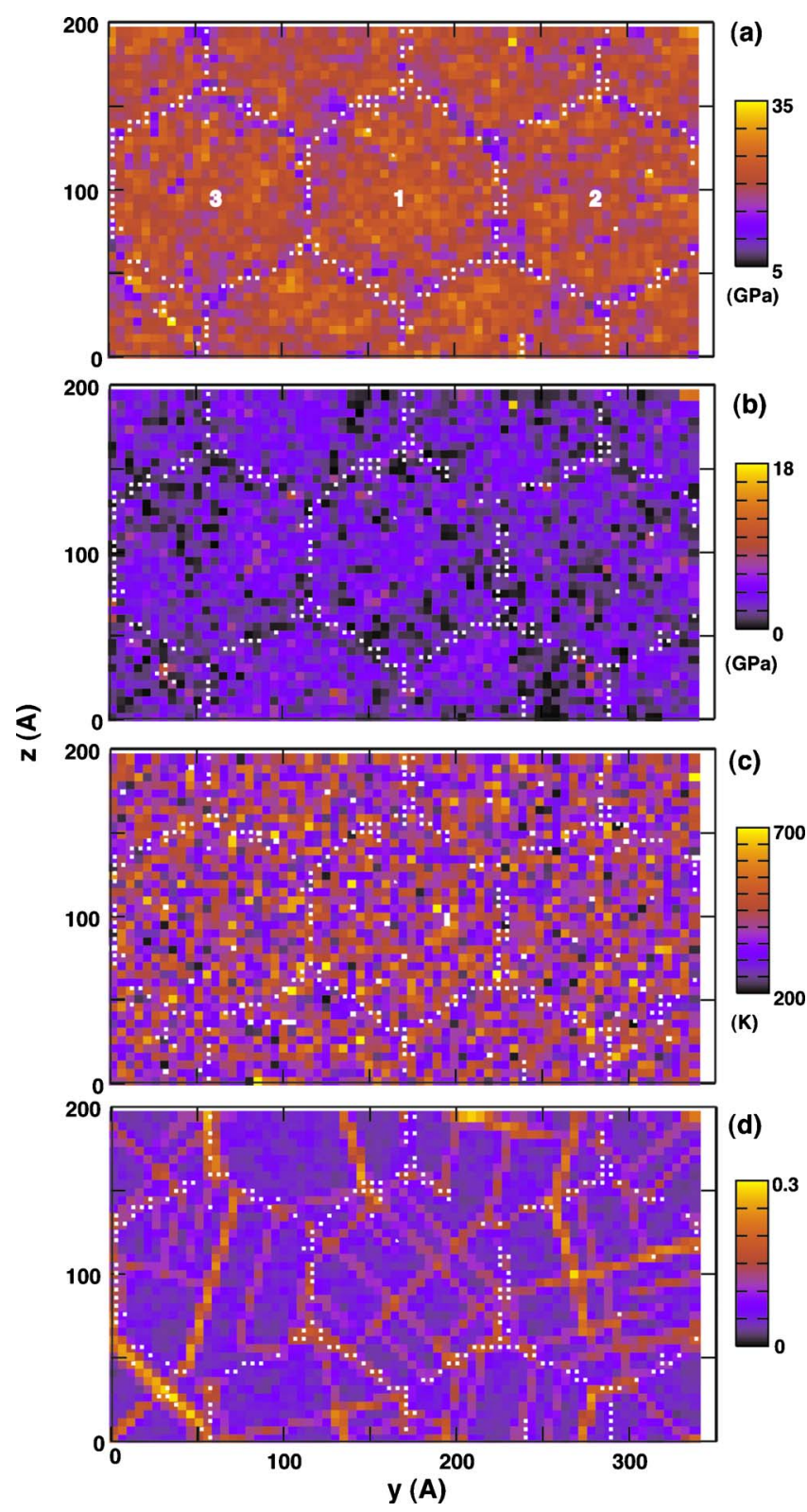

FIG. 7. (Color online) 2D distribution of $\sigma_{x x}(\mathrm{a}),|2 \tau|$ (b), temperature (c), and $\eta^{\mathrm{vM}}(\mathrm{d})$ at the shock state for the $L$-direction loading $(t=14 \mathrm{ps})$. The averaging thickness along the shock direction $(x)$ is $5 \AA$. Numbers $1-3$ in (a) indicate the three typical grains. The GB profiles are shown in white dots.

nanocrystalline $\mathrm{Cu}$ to shock compression. For the T1-direction and T2-direction loading, shear is concentrated at the GBs (not uniformly among all the GBs, though). However, GB shear concentration is absent for the $L$-direction loading. It is simply because the compression loading traverses grains of different mechanical properties for the T1-direction and T2-direction loading; this leads to subsequent grain distortion and thus shear or GB sliding as observed previously in nonshock loading, a result of geometric accommodation. ${ }^{4,12}$ In contrast, all the grains are largely the same with respect to the loading direction for the $L$-direction loading, inducing negligible differential displacements (or misfit in geometry) between neighboring grains and thus little GB shear or sliding. Within the grains, shear deformation is small and does not manifest any well defined shear pattern for the T1-direction and T2-direction loading; how- 

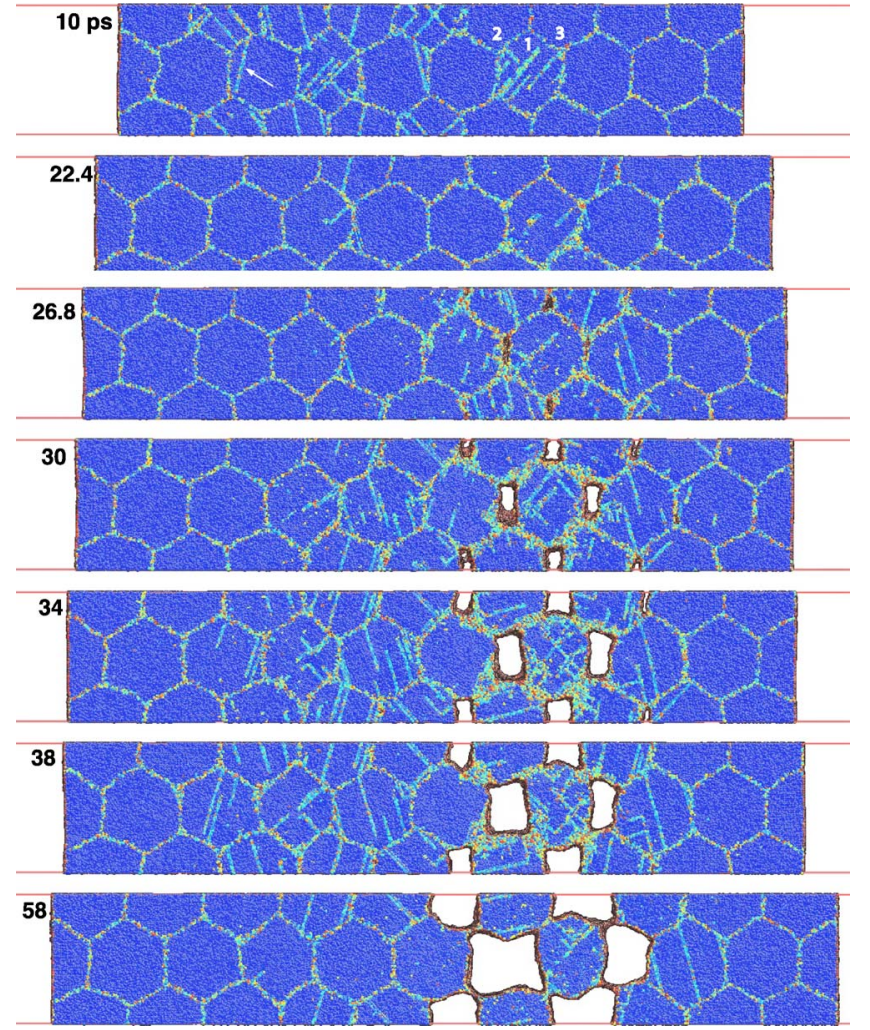

FIG. 8. (Color online) Atomic configurations during shock ( $t=10 \mathrm{ps}$ ), release, tension, and spall for the T1-direction loading. Color coding is based on centrosymmetry. Crystal plasticity (e.g., stacking faults) is indicated with an arrow, and three typical grains (1-3) are noted in the $t=10 \mathrm{ps}$ configuration.

ever, it is pronounced and shows marked slip bands for the $L$-direction loading. Local structure changes during plastic deformation are a direct result of shear, and as expected, the shear characteristics (Figs. 5-7) within the grains are nearly identical to the structural changes (Figs. 8-10). GB sliding may yield apparent plasticity despite the lack of pronounced crystal plasticity for the transverse loading, similar to a MD study on columnar $\mathrm{Al}^{12}$

Both crystal plasticity related shear and GB sliding induce local heating. Heating at GBs is pronounced for the T1-direction and T2-direction loading (strong GB sliding) but minor for the $L$-direction loading (weak GB sliding), and the "hot spots" correlate well with the high shear regions of the GBs. This correlation is vague for heating along the slip bands within the grains, likely due to the low efficiency of heat conversion; the other large portion is consumed as potential energy in inducing, e.g., the fcc-hcp structure change, while such mechanism is absent at GBs so the heat conversion efficiency is much higher. The GB hot spot features are smeared at later times during further thermal and stress relaxations.

From the unshocked to plastic shock region, there is a transition from low to high GB sliding, crystal plasticity, or temperature. Similarly, the elastic-plastic or two-wave structure near the shock front can be identified in the $2 \mathrm{D}$ or $3 \mathrm{D}$ stress profiles as in the 1D analysis (Figs. 5-7; the elastic portion is not shown in Fig. 7 for the L-direction loading). The elastic shock front shows increasing roughening in the

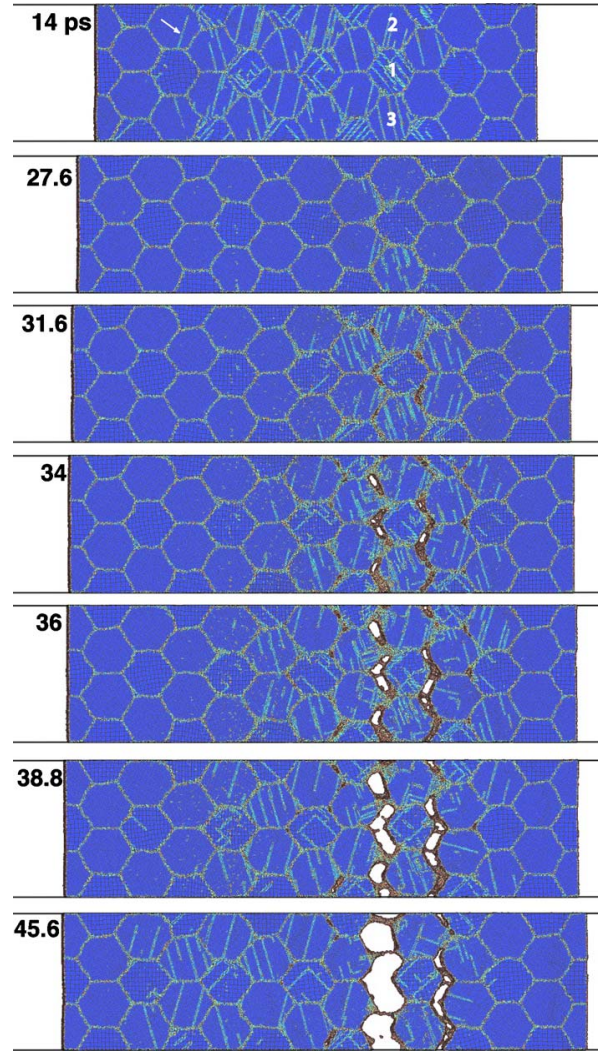

FIG. 9. (Color online) Atomic configurations during shock ( $t=14 \mathrm{ps})$, release, tension, and spall for the T2-direction loading. Also see Fig. 8.

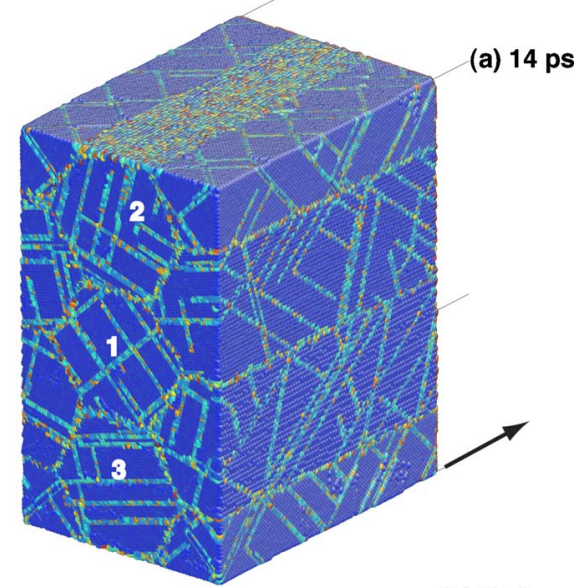

(b) 37.2 ps

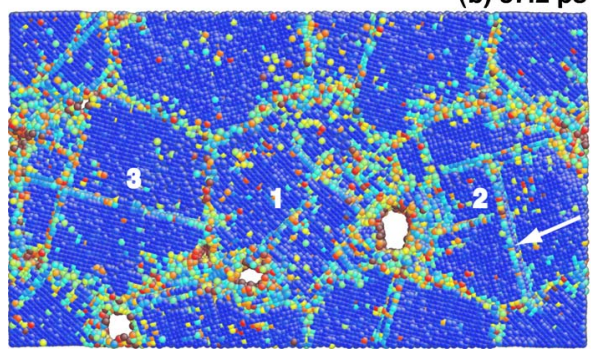

FIG. 10. (Color online) Atomic configurations during shock (a), tension, and spall (b) for the $L$-direction loading. Also see Fig. 8. The arrow in (a) denotes the shock direction, which is out of the paper plane in (b). 

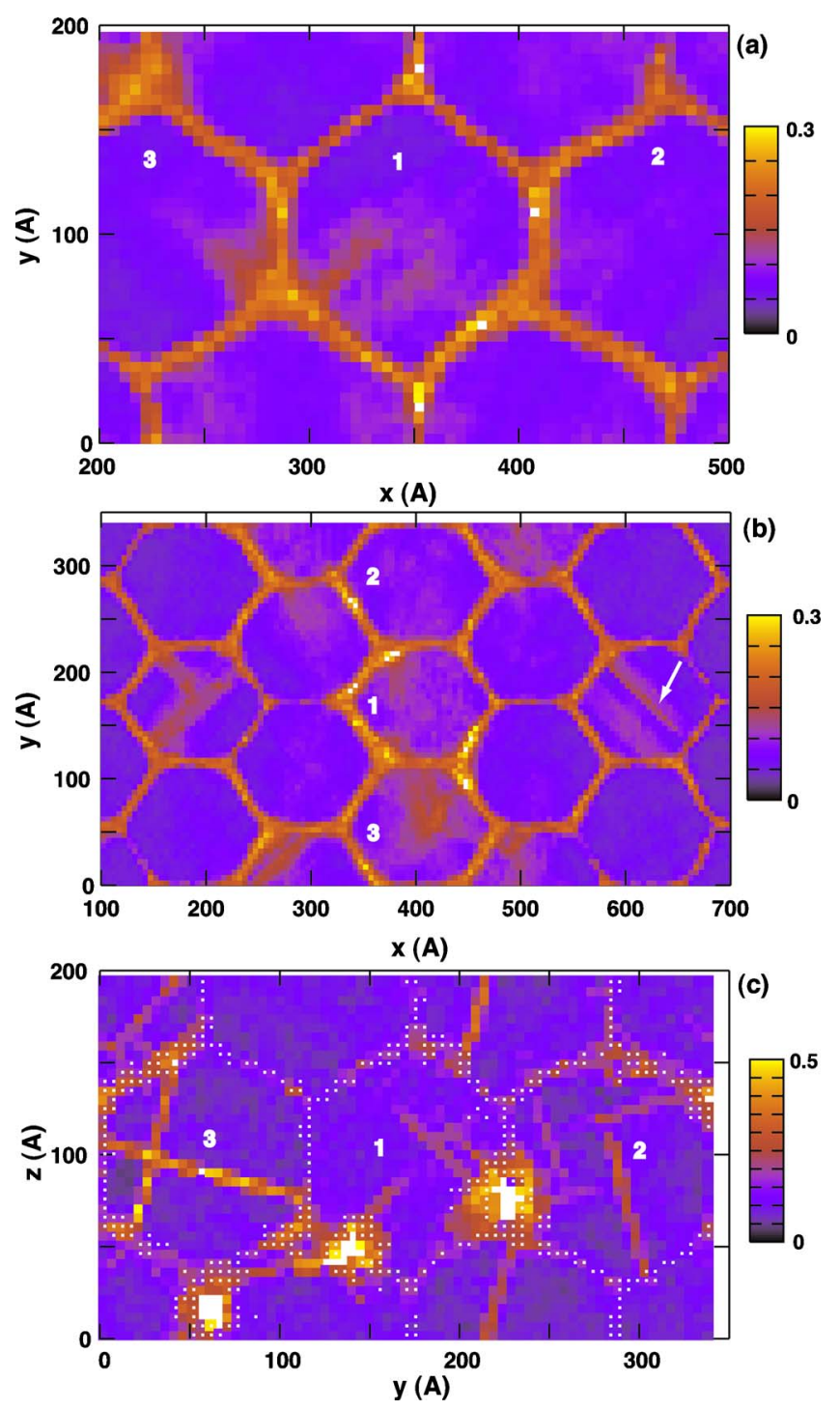

FIG. 11. (Color online) 2D distribution of $\eta^{\mathrm{vM}}$ during tension and void nucleation. (a)-(c) denote the $T 1$-direction, $T 2$-direction, and $L$-direction loading, at $t=26 \mathrm{ps}, 30.8 \mathrm{ps}$, and $37.2 \mathrm{ps}$, respectively. In (a) and (b), averaging along the $L$-direction is adopted, and the averaging thickness along the shock direction $(x)$ is $5 \AA$ in (c). Numbers 1-3 indicate the three typical grains, and the arrow denotes a slip band.

order of the $L$-direction, $T 1$-direction, and $T 2$-directions [Figs. 5-7]. This can simply be explained with the elasticity anisotropy, i.e., different effective wave speeds along the shock direction due to different grain configurations. The wave speed is nearly uniform (except for a slight decrease near the narrow GB regions) along the $L$-direction. For the T2-direction, it is slowest across the centers of type 1 grains while it is fastest across the centers of type 2 or type 3 grains, since the wave speed is slowest along $\langle 100\rangle$; the wave speeds off the grain centers are intermediate between the highest and lowest values, resulting in the pronounced roughening. For the $T 1$-direction, the wave speed represents the average of grain types 1-3 and thus largely depends on the GB regions the wave traverses; as a result, it shows roughening intermediate between the $L$-direction and T2-direction. Roughening of the plastic wave front is also observed for the $T 1$ - and T2-directions, due to both the elasticity anisotropy and plasticity anisotropy, since GB sliding and crystal plasticity may be different as the wave fronts traverse different grains (Figs. 5, 6, 8, and 9).

The GBs induce spatial variations in stresses, e.g., $\sigma_{x x}$ and $|2 \tau|$, during shock compression (Figs. 5-7). For the $T 1$-direction, $\sigma_{x x}$ is reduced at the $l$-type and $m$-type GBs but mostly elevated at the $k$-type GBs, and the opposite occurs for the T2-direction. $\sigma_{x x}$ is reduced at all the GBs for the $L$-direction. Therefore, $\sigma_{x x}$ is reduced if the shock direction lies in the GB plane, and enhanced, if the shock is normal to the GB plane; it can be reduced or enhanced for other orientations. There exist strong stress gradients (positive and negative) as in $\sigma_{x x}$ near the GBs that give rise to the pronounced GB sliding for the T1-direction and T2-direction loading (and opposite for the $L$-direction). $|2 \tau|$ is reduced at all the GBs for all the loading directions (the reduction is minimal for the $L$-direction). This reduction can be directly correlated with such grain movement as sliding or distortion, i.e., the local shear stresses are more prone to relaxation at GBs than within grain interiors. The grain interiors immediate adjacent to the GBs show pronounced stress concentration in $|2 \tau|$ for the transverse loading in type 2 and 3 grains [Figs. 5(b) and 6(b); the stress concentrations are more readily relaxed in type 1 grains via crystal plasticity]. Since relaxation in $|2 \tau|$ within a grain is related to the shear flow stress of a single crystal, it may be different among different grains, and can also be directly correlated with crystal plasticity and associated shear discussed above.

The shocks are reflected at the free surfaces as release fans, which unload the shocked materials to zero stresses (and lower temperature) and further into tension and spall. The structure and shear deformation are characterized for all three loading directions, and Figs. 8-11 show the results during tension and spall. The crystal plasticity induced by shock compression is not stable upon release (partially or completely reversed) due to the lack of effective dislocationpinning mechanisms, for the grain size and loading conditions explored here. Under sufficient tensile loading, plasticity renucleates at GBs (or grows from the remnant plasticity) and progresses with increasing tensile load in the early stage of void nucleation and growth (see below), but diminishes as the void growth unloads tension at later stages. Accompanying well defined crystal plasticity, local disordering is observed within grains or near GBs [e.g., Fig. 10(b)]. The shear deformation within grains reduces during release and rebuilds as tensile loading progresses as well. The slip bands during tension may be reduced due to stress relaxation near the voids. The slip bands within a grain also correspond to the fcc-hcp structure changes [e.g., cf. Figs 10(b) and 11(c)]. Compared to shock compression, the GB sliding is more uniform during tension for the transverse loading, and the amplitude is higher for all the loading directions. Highest shear deformation occurs at GBs, in particular the triple junctions (Fig. 11).

Spallation is essentially a void nucleation and growth process. The spatially resolved measurements are obtained on density, stress, temperature and potential energy at the early stage of void nucleation and growth (Figs. 12-14), besides the structural and shear characteristics presented above 

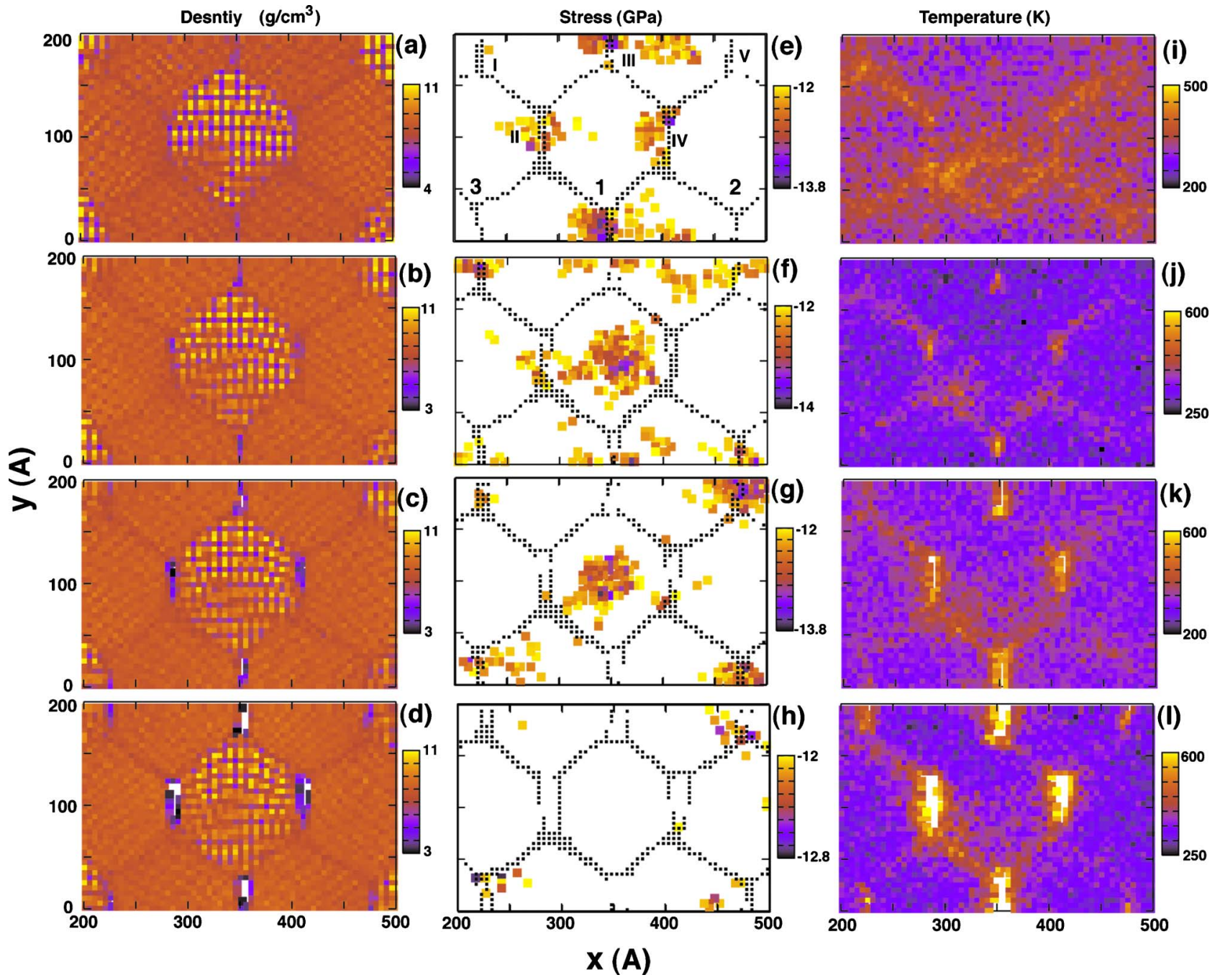

FIG. 12. (Color online) 2D distribution of density (a)-(d) averaged over the $L$-direction, and the corresponding distributions of $\sigma_{x x}$ (e)-(h) and temperature (i)-(1) for the T1-direction loading during void nucleation at GBs. $t=25.2 \mathrm{ps}, 26.0 \mathrm{ps}, 26.8 \mathrm{ps}$, and $27.6 \mathrm{ps}$ for (a)-(d), respectively, as well as for (e)-(h) and (i)-(1). The black dots in (e)-(h) denote the GB profile; numbers 1-3 in (e) indicate three representative grains and, I-V, the $k$-type GBs.

(Figs. 8-11). In Figs. 12(e)-12(h) and 13(e)-13(h), only the highest tensile stress locations are shown for clarity. Characteristic of void nucleation are reduced local density, elevated local temperature and potential energy, and relaxed tensile stress (Figs. 12-14). For the T1-direction loading, tensile stress concentrations occur along the $k$-type GBs besides within grains, and voids only nucleate at such GBs but near the triple junctions (Fig. 12). For the T2-direction loading, tensile stress concentrations are observed along the $l$-type and $m$-type GBs and triple junctions (as well as within grains), and voids only nucleate at such GBs and mostly at the triple junctions (Fig. 13). For the $L$-direction loading, nucleation also occurs at GBs and triple junctions but does not favor any particular GB types (Fig. 14). Void nucleation involves pronounced local disordering (i.e., atoms with low centrosymmetry parameters) at the GBs and triple junctions as seen from the structure analyses (Figs. 8-10). The enhanced shear and heating at GBs and triple junctions also facilitate void nucleation at such locations (Fig. 11).

Void nucleation occurs preferentially at GBs and the triple junctions in particular for spall damage of the columnar $\mathrm{Cu}$, due to their weakened spall strength coupled with stress and shear concentration, for the grain size and loading conditions attempted here. Although some grain interiors undergo higher tensile stresses, no void nucleation is observed because of their higher spall strength (e.g., Figs. 12 and 13). In contrast, crystal plasticity plays the critical role in void nucleation in shocked single crystals; e.g., void nucleates at the cross-section of slip bands, where the pronounced shear and disordering induces local softening. ${ }^{19,20,25}$

Spallation progresses via void growth and coalescence (e.g., Fig. 9). For the T1-direction loading, voids grow along the $k$-type GBs first and then extend into the l-type and $m$-type GBs, while the growth follows the $l$-type and $m$-type GBs but does not extend into the $k$-type GBs for the T2-direction (Figs. 8 and 9). No transgranular damage is induced for the transverse loading. For the $L$-direction, the early growth is along GBs without preferred GB types, but a void nucleated at GBs may grow into grain interiors [the arrow in Fig. 14(c)]. Similar to the void growth within a single crystal, ${ }^{5,20}$ the early stage of the void growth at GBs also involves crystal plasticity as suggested previously. ${ }^{37}$ However, the crystal plasticity can be reduced as local stress 

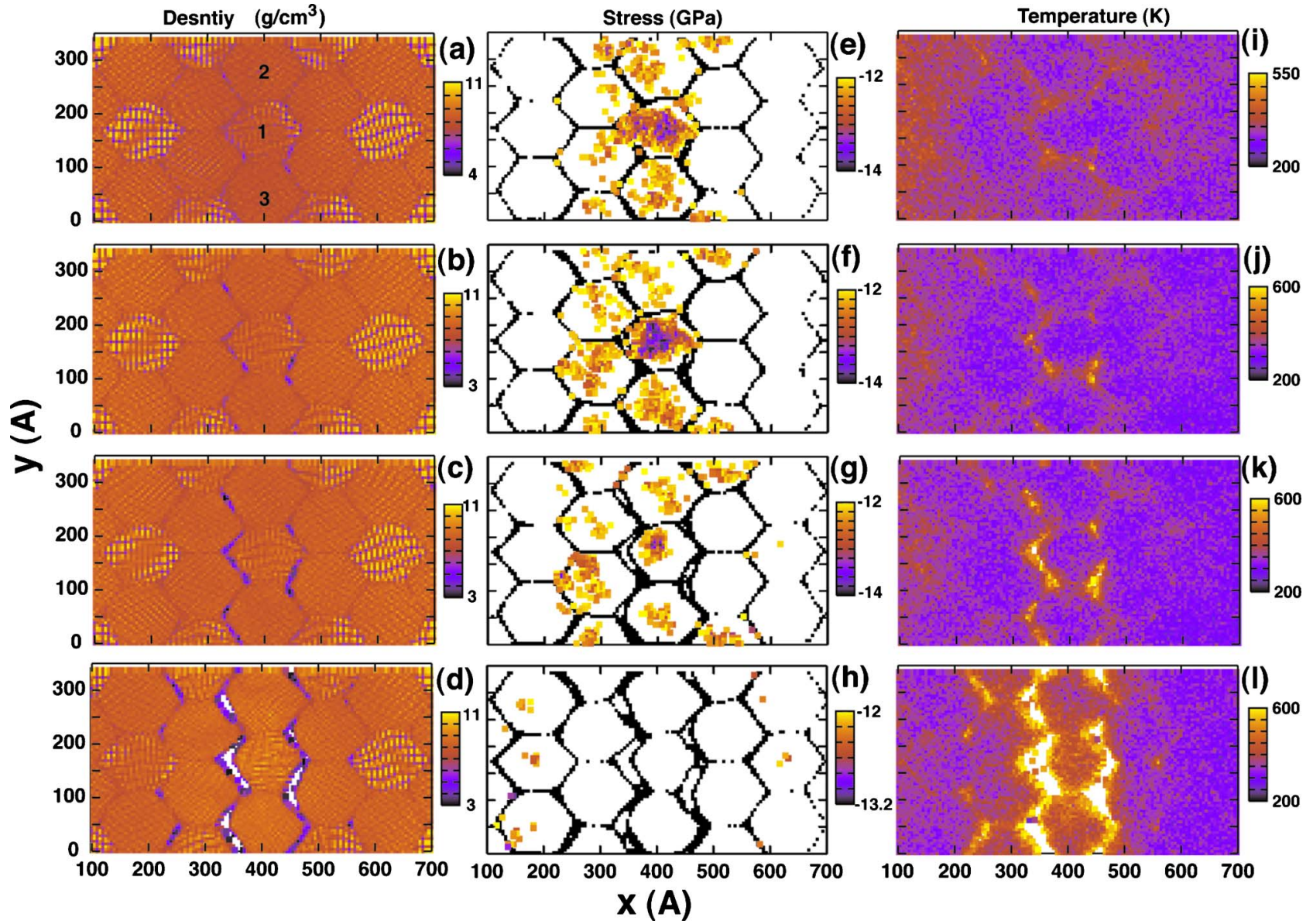

FIG. 13. (Color online) 2D distribution of density (a)-(d) averaged over the $L$-direction, and the corresponding distributions of $\sigma_{x x}$ (e)-(h) and temperature (i)-(1) for the T2-direction loading during void nucleation at GBs. $t=30.8 \mathrm{ps}, 31.6 \mathrm{ps}, 32.8 \mathrm{ps}$, and $34 \mathrm{ps}$ for (a)-(d), respectively, as well as for (e)-(h) and (i)-(1). Numbers $1-3$ in (a) indicate three typical grains. GBs are illustrated in (e)-(h) as black dots.

is relaxed; further growth in such cases as the $T 1$-direction and $T 2$-direction loading is simply achieved via the separation of weak GBs.

The detailed, spatially resolved analysis above points to the inadequacy of 1D analysis and modeling in describing the mechanical behavior of nanocrystalline solids. For example, the stress and shear concentrations near GBs and triple junctions during tension are certainly key to void nucleation but inevitably missing in $1 \mathrm{D}$ modeling. The above analysis also reveals the important role of GBs and triple junctions in compressional and tensile plasticity besides void nucleation and growth, notably, GB sliding, stress, and shear concentration, GB-initiated crystal plasticity, and GB separation. These deformation mechanisms are consistent with previous experiments and simulations under nonshock loading, ${ }^{4,12,13}$ and are valuable for constructing dynamic deformation models of nanocrystalline solids. It is encouraging that recent 2D continuum mechanics modeling revealed similar plastic strain concentration at GBs, ${ }^{13,28,38}$ and MD simulations along this line can help refine 2D or 3D phenomenological modeling at the continuum level.

Both plasticity and spallation behavior of columnar $\mathrm{Cu}$ under shock wave loading depend on grain size and strain rate. Intragranular void nucleation is preceded by intragranular plasticity nucleation; e.g., voids nucleate at intersections of slip bands. ${ }^{20,25}$ Homogeneous nucleation (crystal plasticity and thus voids) is favored by larger grain size and higher strain rates, as opposed to heterogeneous nucleation at GBs. The grain size dependence of the flow stress, or the HallPetch effect and its inverse, was investigated extensively ${ }^{4,12,21}$ but is unclear for columnar solids under shock loading. The crossover in grain size and strain rate for intergranular and transgranular damage under shock loading is underexplored overall. The shock response of columnar solids is expected to depend on the GB energy and shape, rotation axis, and the number of grains. These issues will be a subject of future endeavors beyond the model system explored here.

\section{CONCLUSIONS}

Our results show that the shock response of idealized hexagonal columnar nanocrystalline $\mathrm{Cu}$ is highly anisotropic in local shear, crystal plasticity, and spall damage, and reveal such deformation mechanisms as GB sliding, stress and shear concentration, GB-initiated crystal plasticity, and GB separation. GBs serve as the nucleation sites for crystal plasticity and voids, due to the GB weakening effect as well as stress and strain concentrations. Stress gradients induce GB sliding which is pronounced for the transverse loading. The flow stress and GB sliding are the lowest but the spall strength is the highest for the longitudinal loading. For the 


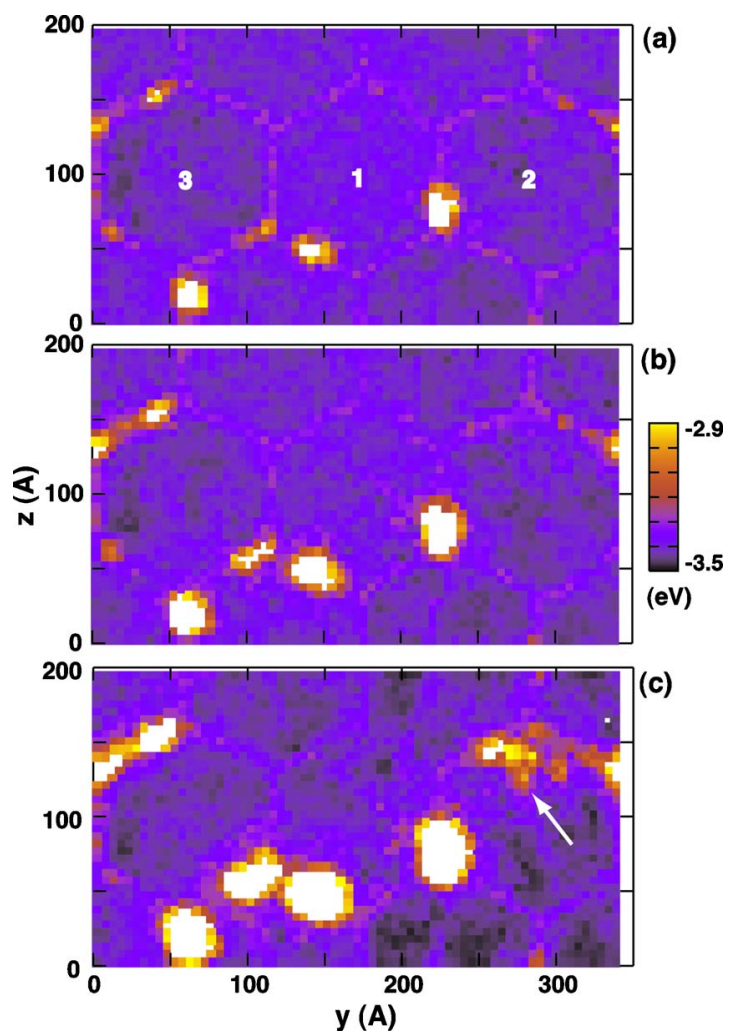

FIG. 14. (Color online) 2D distribution of the potential energy during void nucleation and growth for the $L$-direction loading. (a)-(c) denote $t$ $=37.2 \mathrm{ps}, 38 \mathrm{ps}$, and $39.2 \mathrm{ps}$, respectively. Numbers $1-3$ indicate the three typical types of grains. The averaging thickness along the shock direction $(x)$ is $5 \AA$.

grain size and loading conditions explored, void nucleation occurs at highest shear deformation sites at GBs and in particular the triple junctions; spall damage is entirely intergranular for the transverse loading, while it may extend into grain interiors for the longitudinal loading. Crystal plasticity assists the void growth at the early stage, but the growth is mainly achieved via GB separation at later stages for the transverse loading. These results underscore the necessity of $2 \mathrm{D}$ or $3 \mathrm{D}$ treatment of shock loading of nanocrystalline solids.

\section{ACKNOWLEDGMENTS}

This work is supported by the Laboratory Directed Research and Development (LDRD) program at Los Alamos National Laboratory (Project No. LDRD-20090035DR). LANL is operated by Los Alamos National Security, LLC for the U.S. Department of Energy under Contract No. DEAC52-06NA25396. We have benefited from valuable discussions with A. Koskelo, P. Peralta, B. Holian, B. Uberuaga, C. Barnes, and other colleagues.

${ }^{1}$ J. C. M. Li, Microstructure and Properties of Materials (World Scientific, Singapore, 2000).

${ }^{2}$ M. A. Meyers and C. T. Aimone, Prog. Mater. Sci. 28, 1 (1983).
${ }^{3}$ G. I. Kanel, Int. J. Fract. (unpublished).

${ }^{4}$ K. S. Kumar, H. Van Swygenhoven, and S. Suresh, Acta Mater. 51, 5743 (2003).

${ }^{5}$ M. A. Meyers, S. Traiviratana, V. A. Lubarda, D. J. Benson, and E. M. Bringa, JOM 61, 35 (2009).

${ }^{6}$ A. Hasnaoui, H. Van Swygenhoven, and P. M. Derlet, Phys. Rev. B 66, 184112 (2002).

${ }^{7}$ T. G. Desai, P. C. Millett, and D. Wolf, Acta Mater. 56, 4489 (2008).

${ }^{8}$ T. G. Desai and B. P. Uberuaga, Scr. Mater. 60, 878 (2009).

${ }^{9}$ S. N. Luo, L. Q. Zheng, Q. An, and S. J. Zhao, Int. J. Mod. Phys. C 17, 1551 (2006).

${ }^{10}$ D. Farkas, Metall. Mater. Trans. A 38, 2168 (2007).

${ }^{11}$ D. Farkas and W. A. Curtin, Mater. Sci. Eng., A 412, 316 (2005).

${ }^{12}$ V. Yamakov, D. Wolf, M. Salazar, S. R. Phillpot, and H. Gleiter, Acta Mater. 49, 2713 (2001).

${ }^{13}$ L. Anand and Y. J. Wei, in IUTAM Symposium on Mechanical Behavior and Micro-Mechanics of Nanostructured Materials, edited by Y. L. Bai, Q. S. Zheng, and Y. G. Wei (Springer, Berlin, 2007), p. 3.

${ }^{14}$ C. H. Xiao, R. A. Mirshams, S. H. Whang, and W. M. Yin, Mater. Sci. Eng., A 301, 35 (2001).

${ }^{15}$ R. J. Asaro, P. Krysl, and B. Kad, Philos. Mag. Lett. 83, 733 (2003).

${ }^{16}$ B. L. Holian, Shock Waves 5, 149 (1995).

${ }^{17}$ T. C. Germann, B. L. Holian, P. S. Lomdahl, and R. Ravelo, Phys. Rev. Lett. 84, 5351 (2000).

${ }^{18}$ A. Kubota, D. B. Reisman, and W. G. Wolfer, Appl. Phys. Lett. 88, 241924 (2006).

${ }^{19}$ Y. Ashkenazy and R. S. Averback, Appl. Phys. Lett. 86, 051907 (2005).

${ }^{20}$ S. N. Luo, T. C. Germann, and D. L. Tonks, J. Appl. Phys. 106, 123518 (2009).

${ }^{21}$ E. M. Bringa, A. Caro, Y. Wang, M. Victoria, J. M. McNaney, B. A. Remington, R. F. Smith, B. R. Torrala, and H. Van Swygenhoven, Science 309, 1838 (2005).

${ }^{22}$ V. Dremov, A. Petrovtsev, P. Sapozhnikov, M. Smirnova, D. L. Preston, and M. A. Zocher, Phys. Rev. B 74, 144110 (2006).

${ }^{23}$ G. E. Norman, A. Y. Kuksin, V. V. Stegailov, and A. V. Yanilkin, in Shock Compression of Condensed Matter-2007, edited by M. Elert, M. D. Furnish, R. Chau, N. Holmes, and J. Nguyen (American Institute of Physics, Melville, 2008), Vol. CP955, p. 329.

${ }^{24}$ S. G. Srinivasan, M. I. Baskes, and G. J. Wagner, J. Appl. Phys. 101, 043504 (2007)

${ }^{25}$ S. N. Luo, T. C. Germann, and D. L. Tonks, J. Appl. Phys. 107, 056102 (2010).

${ }^{26}$ D. L. Paisley, S. N. Luo, S. R. Greenfield, and A. C. Koskelo, Rev. Sci. Instrum. 79, 023902 (2008).

${ }^{27}$ P. Peralta, S. DiGiacomo, S. Hashemian, S. N. Luo, D. Paisley, R. Dickerson, E. Loomis, D. Byler, and K. J. McClellan, Int. J. Damage Mech. 18, 393 (2009).

${ }^{28}$ D. L. Tonks, D. L. Paisley, P. D. Peralta, S. R. Greenfield, D. D. Byler, S. N. Luo, D. C. Swift, and A. C. Koskelo, in Shock Compression of Condensed Matter-2007, edited by M. Elert, M. D. Furnish, R. Chau, N. Holmes, and J. Nguyen (American Institute of Physics, Melville, 2008), Vol. CP955, p. 605.

${ }^{29}$ Y. Mishin, M. J. Mehl, D. A. Papaconstantopoulos, A. F. Voter, and J. D. Kress, Phys. Rev. B 63, 224106 (2001).

${ }^{30}$ J. Stadler, R. Mikulla, and H.-R. Trebin, Int. J. Mod. Phys. C 8, 1131 (1997).

${ }^{31}$ A. Okabe, B. Boots, K. Sugihara, and S. N. Chiu, Concepts and Applications of Voronoi Diagrams, 2nd ed. (Wiley, New York, 2000).

${ }^{32}$ S. N. Luo, Q. An, T. C. Germann, and L. B. Han, J. Appl. Phys. 106, 013502 (2009).

${ }^{33}$ J. F. Lutsko, J. Appl. Phys. 65, 2991 (1989).

${ }^{34}$ F. Shimizu, S. Ogata, and J. Li, Mater. Trans. 48, 2923 (2007).

${ }^{35}$ C. L. Kelchner, S. J. Plimpton, and J. C. Hamilton, Phys. Rev. B 58, 11085 (1998).

${ }^{36}$ J. Li, Modell. Simul. Mater. Sci. Eng. 11, 173 (2003).

${ }^{37}$ J. N. Johnson, J. Appl. Phys. 52, 2812 (1981).

${ }^{38}$ Y. J. Wei and L. Anand, J. Mech. Phys. Solids 52, 2587 (2004). 